\title{
Decreased blood CD4+PD-1+ and CD8+PD-1+ T cells in psoriatic patients with and without arthritis
}

\author{
Joanna Bartosińska', Ewelina Zakrzewska², Joanna Purkot² ${ }^{2}$ Anna Michalak-Stoma ${ }^{1}$, Małgorzata Kowal', \\ Dorota Krasowska ${ }^{1}$, Grażyna Chodorowska ${ }^{1}$, Krzysztof Giannopoulos ${ }^{2}$
}

${ }^{1}$ Department of Dermatology, Venereology and Paediatric Dermatology, Medical University of Lublin, Lublin, Poland Experimental Hematooncology Department, Medical University of Lublin, Lublin, Poland

Adv Dermatol Allergol 2018; XXXV (4): 344-350

DOI: https://doi.org/10.5114/ada.2018.75609

\begin{abstract}
Introduction: Psoriasis with and without arthritis have common immunological mechanisms which among others involve the interactions between cytokines produced by T cells, including Th1, Th17 and Th22. Although quite a lot is known about psoriasis pathogenesis, the cause of chronic immune activation and response in the disease remains unclear. One of the negative regulators of the immune system is programmed death 1 (PD-1).

Aim: To assess the expression level of PD-1 in the peripheral T cells of psoriatic patients with and without arthritis. Material and methods: The study included 23 psoriatic patients with arthritis, 52 psoriatic patients without arthritis and 52 healthy controls. The percentages of CD3+, CD4+, CD8+, CD4+PD-1+ and CD8+PD-1+ T cells were analyzed using flow cytometry.

Results: The percentages of CD4+PD-1+ as well as CD8+PD-1+ T cells in the psoriatic patients both with and without arthritis were significantly lower than in the control group. The percentages of CD4+PD-1+ as well as CD8+PD-1+ $T$ cells were not significantly different between the psoriatic patients with and without arthritis. A significant positive correlation between PD-1 expression on the CD4+ and CD8+ T cells was found in the psoriatic patients without arthritis.

Conclusions: Impairment of the negative co-stimulation from PD-1 may be another common characteristic of psoriasis both with and without arthritis.
\end{abstract}

Key words: psoriasis, psoriatic arthritis, programmed death 1.

\section{Introduction}

An erythematous plaque covered with silvery scales is a typical skin lesion of psoriasis, a chronic systemic inflammatory disease which presents itself in various clinical forms. The disease is heterogeneous, and whether it will assume a mild or more severe course may depend on genetic variants of psoriasis [1]. Even though in about $30 \%$ of psoriatic patients psoriatic arthritis (PsA) is also diagnosed, it is debatable whether PsA may be regarded as one of the psoriasis manifestations or whether it is a separate and independent form of arthritis. These two types of psoriasis, i.e. psoriasis with and without arthritis, share plenty of immunological mechanisms, yet their pathogenesis has not been fully explained so far. It is thought that innate immunity plays a certain role in psoriasis initiation with a subsequent response of various T cell subsets, not only Th1 but also Th17, Th22 and their cytokines, which will ultimately lead to formation of psoriasis auto-inflammatory pathways [2]. Nevertheless, the cause of immune imbalance and chronic activation of the T cell subsets in psoriasis should be further investigated. The recently published reports indicate that during anti-programmed death 1 (PD-1) and anti-cytotoxic T-lymphocyte-associated protein 4 (CTLA-4) therapies, skin and articular complications, including psoriasis, may occur; therefore, it seems plausible that negative T cell co-stimulation may be impaired in psoriasis [3-5]. In experimental studies PD-1 $1^{--}$T cells were able to produce increased amounts of interferon $\gamma($ IFN- $\gamma)$ and interleukin (IL)-17, thereby confirming the key role of PD-1 in the regulation of Th1 and Th17 responses [6]. In order to detect immunomodulatory agents capable of reducing an inflammatory process in the course of psoriasis, an in-depth investigation of immune tolerance mecha-

Address for correspondence: Joanna Bartosińska MD, PhD, Department of Dermatology, Venereology and Paediatric Dermatology, Medical University of Lublin, 13 Radziwiłłowska St, 20-080 Lublin, Poland, phone: +48 602724 298, e-mail: jbartosinski@gmail.com Received: 31.05.2017, accepted: 15.06.2017. 
nisms in psoriasis, both with and without arthritis, is worth launching.

\section{Aim}

The aim of the study was to assess the expression level of PD-1 in the peripheral T cells of psoriatic patients with and without arthritis.

\section{Material and methods}

\section{Study groups}

The study included 75 patients: 23 (30.67\%) psoriatic patients with arthritis and 52 (69.33\%) psoriatic patients without arthritis hospitalized in the Department of Dermatology, Venereology and Pediatric Dermatology at the Medical University of Lublin, Poland, as well as 52 age- and gender-matched healthy volunteers. Psoriatic arthritis (PSA) was diagnosed using the Classification of Psoriasis Arthritis (CASPAR) criteria.

The inclusion criteria were: the duration of psoriasis for at least one year, active psoriatic skin lesions, age at least 18 years. The exclusion criteria were: cardiovascular, cerebrovascular, hematologic, hepatic or renal disease, neoplasm, chronic viral infections, erythrodermic, pustular or guttate psoriasis, addiction to drugs, systemic anti-psoriatic treatment.

The study was approved by the Local Ethic Committee at the Medical University of Lublin (KE-0254/81/2015). Informed consent was obtained from all the participants.

The study was supported by grant No. 164 of the Medical University of Lublin.

\section{Assessment of psoriasis severity}

The severity of psoriatic skin lesions was assessed with Psoriasis Area and Severity Index (PASI), body surface area (BSA), Patient Global Assessment (PGA), and Dermatology Life Quality Index (DLQI), and nail plate changes with Nail Psoriasis Severity Index 80 (NAPSI 80).

The severity of psoriatic arthritis was assessed with the Disease Activity Score 28 (DAS 28), Patient Visual Analog Scale (VAS), number of tender joints, number of swollen joints, and physician and patient Likert scales.

In studied patients laboratory tests, i.e. blood morphology, C-reactive protein (CRP), and (erythrocyte sedimentation rate (ESR), were performed in the hospital central laboratory.

\section{Flow cytometry analysis of peripheral blood mononuclear cell populations and expression of PD-1}

Flow cytometry analysis was performed in the Experimental Hematooncology Department at the Medical University of Lublin, Poland, with staff professionally trained and experienced in such analyses in patients with lymphoproliferative diseases.

Eight $\mathrm{ml}$ of peripheral venous blood from the psoriatic patients with and without arthritis as well as healthy volunteers were collected into anti-coagulated tubes. We isolated mononuclear cells using density gradient centrifugation on Ficoll-Hypaque (Biochrom AG, Berlin, Germany). Interphase cells were removed, washed twice in phosphate-buffered saline (PBS) without $\mathrm{Ca}^{2+}$ and $\mathrm{Mg}^{2+}$ and resuspended in RPMI 1640 containing 2\% human albumin. The viability of obtained peripheral blood mononuclear cells (PBMCs) was always > 95\%, as determined by trypan blue staining. Viable cells were quantified in a Neubauer chamber. $5 \times 10^{5}$ cells were incubated for $20 \mathrm{~min}$ at room temperature with fluorochrome-labeled monoclonal antibodies (Mabs): anti-CD3-PerCP (Becton Dickinson), anti-CD4-FITC (Becton Dickinson), anti-CD8PE (Becton Dickinson) and anti-PD-1-APC (clone MIH4) (Becton Dickinson). Approximately 100,000 stained cells in each sample were analyzed by flow cytometry using a FACS Canto II flow cytometer (BD Biosciences, San Jose, (A, USA). Unstained cells were used as a negative control (for each patient). Data analysis was accomplished by FACS Diva 8.0.

For each person, lymphocytes were identified and gated from PBMCs by setting appropriate forward and side scatter parameters. Next, 3 steps of gating were applied in flow cytometry analysis. The first dot plot showed CD3 + cells selected from all lymphocytes and as a result we obtained percentages of CD3+ cells of all lymphocytes. The two gates at the second step of the cytometry selected: one gate CD4+ and the other gate CD8+ T cells separately, resulting in percentages of CD4+ and CD8+ T cells, respectively, of all CD3+ cells. The two gates at the third step of the cytometry selected: one gate CD4+PD-1+ $T$ cells and the other gate CD8+PD-1+ $T$ cells separately, resulting in percentages of CD4+PD-1+ $T$ cells and CD8+PD-1+ T cells of CD4+ and CD8+ T cells, respectively (Figure 1 shows it for 3 exemplary persons: 1 patient with psoriatic arthritis, 1 patient with psoriasis without arthritis and 1 healthy volunteer)

\section{Statistical analysis}

Mean values (M) and standard deviations (SD) were calculated for continuous variables, or absolute numbers $(n)$ and relative numbers (\%) of occurrence of items of categorical variables. We used the stochastic independence $\chi^{2}$ test to compare sex between the control group and psoriatic patients with and without arthritis, as well as positive family history of psoriasis of psoriatic patients with and without arthritis. We used the Mann-Whitney $U$ test to compare: age, percentages of PBMCs and PD-1 between the control group and psoriatic patients with and without arthritis, and other clinical data (measured as continuous variables) between psoriatic patients with and without arthritis. 

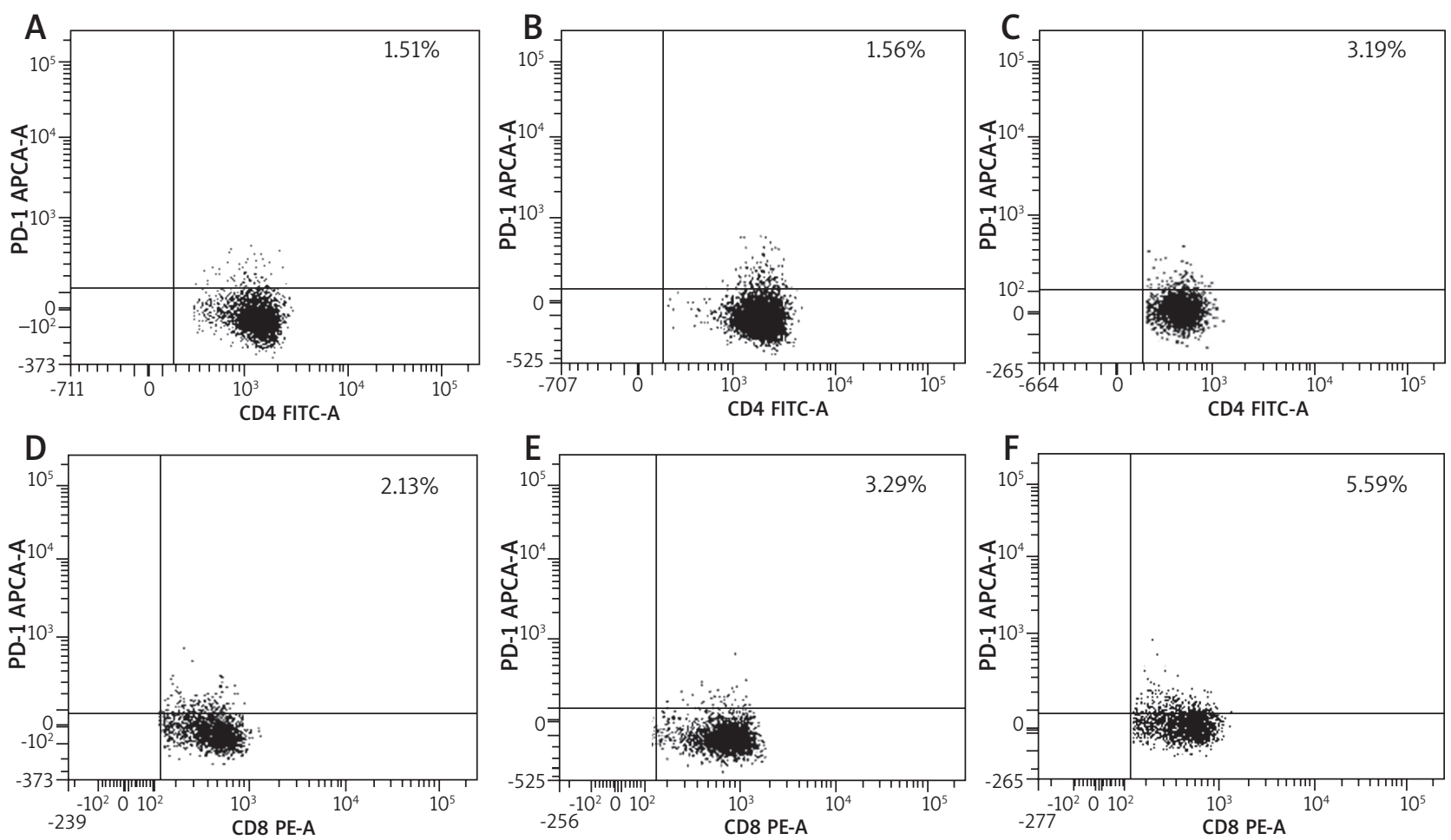

Figure 1. Representative flow cytometry analysis of PD-1 expression on CD4+ (A-C) and CD8+ (D-F) T cells from psoriatic patients without arthritis (A, D), with arthritis (B, E) and healthy controls (C, F)

We used Spearman's correlation coefficient to investigate mutual correlations between the expression of PD-1 on CD4+ and CD8+ T cells and between this expression and clinical data in psoriatic patients, separately with and without arthritis.

Statistical analysis was performed using Statistica software. The significance level was set at 0.05.

\section{Results}

Characteristics of studied psoriatic patients with and without arthritis and healthy controls

Demographic characteristics and clinical data, duration, severity and family history and laboratory blood tests (ESR and CRP) of the patients are described in Table 1. Psoriatic patients with and without arthritis and the control group did not significantly differ in sex $(p<0.05)$. In the control group 43 out of 52 were males, that is $82.69 \%$. Mean age of the control group was $48.7 \pm 15.2$ years and did not significantly differ from patients with and without arthritis $(p<0.05)$. However, patients with arthritis were significantly older than patients without arthritis. Psoriasis duration was significantly longer in psoriatic patients with arthritis compared to patients without arthritis. Psoriasis severity did not significantly differ between psoriatic patients with and without arthritis.
Clinical data and psoriasis arthritis severity are also presented in Table 1: mean duration of PsA 9.3 years, mean age of disease onset 42 years, mean number of tender joints 4.6, mean number of swollen joints 1.3, mean value of VAS 40 , mean value of DAS 3.2. The majority of patients (65.2\%) had the oligoarticular subset of PSA.

\section{Comparison of PBMC populations' distribution between psoriatic patients with and without arthritis and healthy controls}

The percentages of CD3+, but not CD4+ and CD8+, T cells were significantly higher in the psoriatic patients with arthritis in comparison with the control group. The percentages of CD3+ and CD8+, but not CD4+, $T$ cells were significantly higher in the psoriatic patients without arthritis in comparison with the control group. The expression of PD-1 on CD4+ and CD8+ T cells was significantly lower in psoriatic patients with arthritis in comparison with the control group as well as in psoriatic patients without arthritis in comparison with the control group (Table 2).

Percentages of CD3+, CD4+, CD8+, CD4+PD-1+ and CD8+PD-1+ T cells were not significantly different between psoriatic patients with and without arthritis $(p>0.05)$ 
Table 1. Clinical data of psoriatic patients with and without arthritis

\begin{tabular}{|c|c|c|c|}
\hline Clinical data & $\begin{array}{l}\text { Psoriasis with arthritis } \\
\qquad(n=23)\end{array}$ & $\begin{array}{l}\text { Psoriasis without arthritis } \\
\qquad(n=52)\end{array}$ & $P$-value \\
\hline Age, $M \pm S D$ [years] & $53.1 \pm 8.9$ & $44.4 \pm 15.9$ & 0.011 \\
\hline Sex - male, n (\%) & $19(82.6)$ & $46(88.5)$ & 0.500 \\
\hline Duration of psoriasis, $\mathrm{M} \pm \mathrm{SD}$ [years] & $28.8 \pm 11.6$ & $16.1 \pm 12.7$ & $<0.001$ \\
\hline Age of psoriasis onset, $\mathrm{M} \pm \mathrm{SD}$ [years] & $24.3 \pm 9.4$ & $28.3 \pm 16.3$ & 0.573 \\
\hline Positive family history of psoriasis, $n(\%)$ & $12(52.2)$ & $16(30.8)$ & 0.080 \\
\hline $\mathrm{PASI}, \mathrm{M} \pm \mathrm{SD}$ & $15.0 \pm 11.5$ & $13.5 \pm 8.3$ & 0.998 \\
\hline $\mathrm{BSA}(\%), \mathrm{M} \pm \mathrm{SD}$ & $25.3 \pm 18.6$ & $23.4 \pm 17.6$ & 0.877 \\
\hline $\mathrm{PGA}, \mathrm{M} \pm \mathrm{SD}$ & $3.0 \pm 0.8$ & $3.1 \pm 0.8$ & 0.909 \\
\hline $\mathrm{NAPSI}, \mathrm{M} \pm \mathrm{SD}$ & $21.1 \pm 17.2$ & $17.4 \pm 16.2$ & 0.177 \\
\hline $\mathrm{DLQI}, \mathrm{M} \pm \mathrm{SD}$ & $15.7 \pm 7.4$ & $13.3 \pm 8.0$ & 0.323 \\
\hline Erythrocyte sedimentation rate, $M \pm S D[\mathrm{~mm} / \mathrm{h}]$ & $12.1 \pm 8.4$ & $12.2 \pm 12.2$ & 0.346 \\
\hline C-reactive protein, $\mathrm{M} \pm \mathrm{SD}$ [mg/dl] & $4.2 \pm 5.8$ & $3.7 \pm 8.3$ & 0.283 \\
\hline Duration of PsA (psoriatic arthritis), $M \pm S D$ [years] & $9.3 \pm 5.9$ & & \\
\hline Age of PsA onset, $M \pm S D$ [years] & $42.0 \pm 12.1$ & & \\
\hline \multicolumn{4}{|l|}{ Clinical subsets of PsA $n(\%)$ : } \\
\hline Oligoarticular ( $\leq 5$ joints) & $15(65.2)$ & & \\
\hline Polyarticular & $6(26.1)$ & & \\
\hline Distal interphalangeal predominant & $2(8.7)$ & & \\
\hline Spondylitis predominant & $0(0.0)$ & & \\
\hline Mutilans & $0(0.0)$ & & \\
\hline Tender Joint count, $\mathrm{M} \pm \mathrm{SD}$ & $4.6 \pm 3.6$ & & \\
\hline Swollen Joint count, $M \pm S D$ & $1.3 \pm 1.9$ & & \\
\hline 5-point Likert scale (physician), $M \pm S D$ & $2.1 \pm 0.8$ & & \\
\hline 5-point Likert scale (patient), $M \pm S D$ & $2.4 \pm 0.9$ & & \\
\hline Patient Visual Analog Scale (VAS), $M \pm S D$ & $40.0 \pm 25.0$ & & \\
\hline Disease Activity Score (DAS28), $M \pm S D$ & $3.2 \pm 1.1$ & & \\
\hline
\end{tabular}

Table 2. Comparison of the cell populations of PBMCs and PD-1 expression in psoriatic patients with and without arthritis as well as the control group

\begin{tabular}{|c|c|c|c|c|c|c|}
\hline \multirow{2}{*}{$\begin{array}{l}\text { PBMC and PD-1 } \\
\text { expression }\end{array}$} & \multirow{2}{*}{$\begin{array}{l}\text { Psoriasis with } \\
\text { arthritis } \\
\begin{array}{l}(n=23) \\
M \pm S D\end{array}\end{array}$} & \multirow{2}{*}{$\begin{array}{l}\text { Psoriasis } \\
\text { without } \\
\text { arthritis } \\
(n=52) \\
M \pm S D\end{array}$} & \multirow{2}{*}{$\begin{array}{l}\text { Control } \\
(n=52) \\
M \pm S D\end{array}$} & \multicolumn{3}{|c|}{$P$-value } \\
\hline & & & & $\begin{array}{l}\text { Psoriasis with } \\
\text { arthritis versus } \\
\text { control }\end{array}$ & $\begin{array}{l}\text { Psoriasis without } \\
\text { arthritis versus } \\
\text { control }\end{array}$ & $\begin{array}{c}\text { Psoriasis with } \\
\text { arthritis versus } \\
\text { psoriasis without } \\
\text { arthritis }\end{array}$ \\
\hline CD3+ (\%) & $60.1 \pm 12.6$ & $58.8 \pm 13.3$ & $48.2 \pm 17.0$ & 0.002 & 0.001 & 0.694 \\
\hline CD4+ (\%) & $61.4 \pm 19.8$ & $58.5 \pm 17.6$ & $54.0 \pm 17.5$ & 0.143 & 0.257 & 0.533 \\
\hline CD8+ (\%) & $29.6 \pm 18.7$ & $31.7 \pm 16.0$ & $23.3 \pm 10.1$ & 0.405 & 0.011 & 0.623 \\
\hline CD4+PD-1 (\%) & $1.6 \pm 0.7$ & $2.1 \pm 1.3$ & $2.9 \pm 1.7$ & 0.001 & 0.022 & 0.084 \\
\hline CD8+PD-1 (\%) & $3.4 \pm 2.3$ & $3.2 \pm 2.1$ & $6.6 \pm 4.4$ & 0.001 & $<0.001$ & 0.704 \\
\hline
\end{tabular}


A

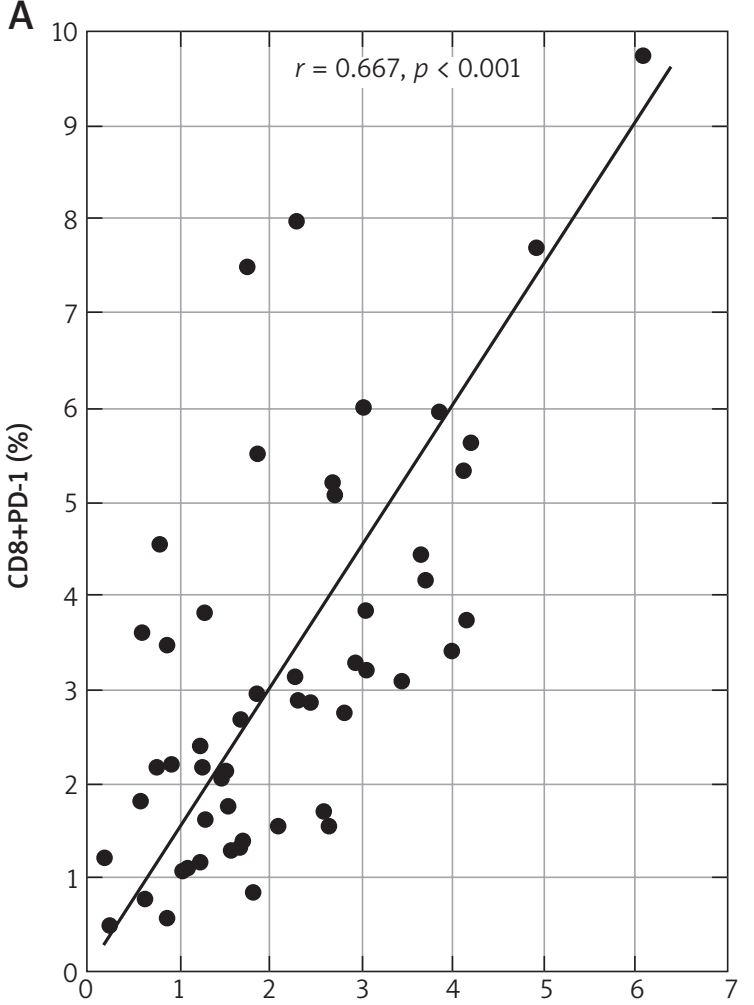

B

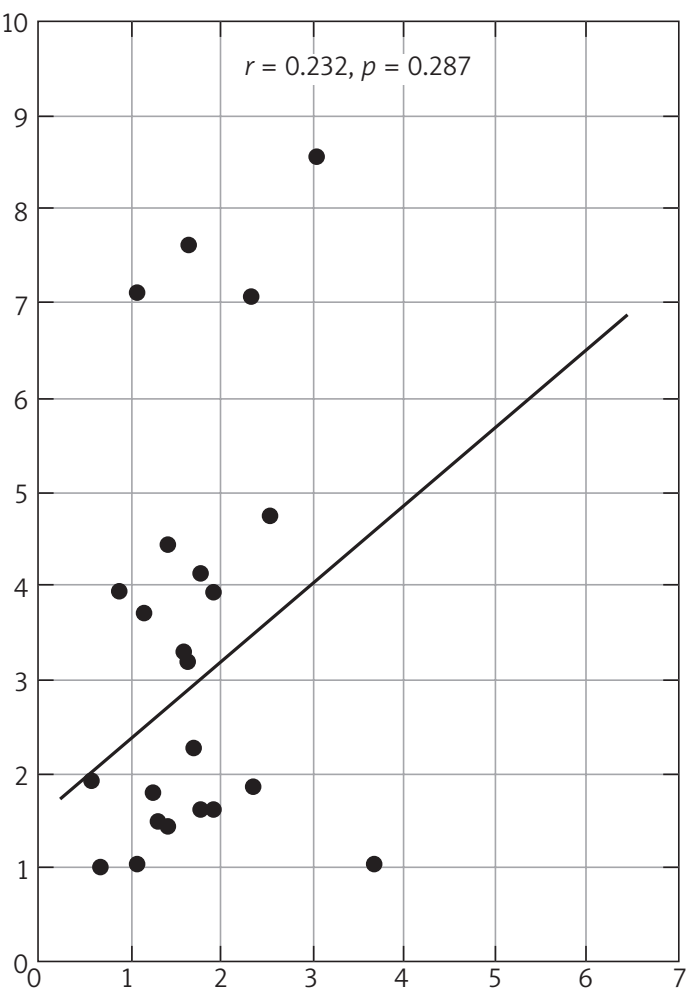

CD4+PD-1 (\%)

Figure 2. Correlation between CD4+PD-1 and CD8+PD-1 for psoriatic patients without (A) and with (B) arthritis

Analysis of mutual correlations between CD4+PD-1+ and CD8+PD-1+ in patients with and without arthritis

The percentages of CD4+PD-1+ correlated positively with the percentages of CD8+PD-1 in patients without arthritis, but not in patients with arthritis (Figure 2).

\section{Analysis of correlations between PBMC}

populations' distribution and clinical data in psoriatic patients with and without arthritis

The expression of PD-1 on CD4+ and CD8+ T cells did not correlate with any of the studied clinical data in the psoriatic patients with and without arthritis $(p>0.05)$.

\section{Discussion}

In our study we assessed the whole spectrum of T cells with respect to PD-1 in the psoriatic patients with and without arthritis in comparison to the control group.

In this study, we provided evidence that the expression of PD-1 on the CD4+ and CD8+ T cells is altered in psoriatic patients; therefore, the PD-1/PDL-1 pathway may be involved in the regulation of T-cell activation and function in psoriatic patients both with and without arthritis. Therefore, the finding may also confirm the concept of "psoriatic disease" and allow psoriasis to be considered as a single entity together with identified comorbidities [7].

In psoriatic arthritis (PSA) chronic inflammation results in enthesis, cartilage and bone damage, leading to joint and bone destruction and remodeling. In the majority of patients a skin manifestation of psoriasis occurs prior to the joint involvement, which may be suggestive of some skin pathological inflammatory processes playing a role in the development of arthritis [8].

Both psoriasis and PsA were initially regarded as Th1mediated diseases, due to differentiation of the naïve T cells after IL-12 and IFN- $\gamma$ release by antigen-presenting cells (APCS). The psoriatic skin and synovium are infiltrated by the T cells, dendritic cells, neutrophils and macrophages. However, it is well known that Th17 cells, activated by IL-23, IL-21, IL- 6 and transforming growth factor- $\beta$ (TGF- $\beta$ ), play a vital role in the pathogenesis of psoriasis and PSA. Th17 cells are present not only in the skin but also in the synovial tissue and synovial fluid $[9,10]$. Some animal studies have revealed that mice deficient in IL-17 showed decreased development of collagen-induced arthritis [11]. Nevertheless, the mechanism of chronic T cell activation by APCs in psoriasis still awaits elucidation. Impaired interaction of the PD-1 on T cells, B cells and macrophages with its ligand PD-L1 or PD-L2 on the APCS seems to be responsible for an intense, up-regulating T cell response [12, 13]. 
In our study psoriatic patients with arthritis constituted $30.67 \%$ of all studied subjects. In the psoriatic patients with arthritis, similarly to the patients without arthritis, the percentages of both CD4+PD-1+ and CD8+PD-1+ T cells were significantly lower than in the control group. This finding is in agreement with the concept of the PD-1/PD-L1 axis in the down-regulating T cell response. The observation may confirm the hypothesis of the convergent role of PD-1 in psoriasis with and without arthritis. However, it is generally assumed that the development of psoriatic changes in the joints, cartilages and tendons may be insidious and some discrete inflammatory processes are slowly progressing, bringing about subclinical changes sometimes hard to detect even by high-tech diagnostic tools.

Peled et al. [14], in 20 PsA patients, found that the percentage of CD3+PD-1+ T cells was higher in patients than healthy controls. However, the authors observed an inverse correlation with disease activity: the higher the number of swollen and/or tender joints, the lower the percentage of PD-1 expressing T cells. In our study we did not find any correlation between the severity of PsA, including the number of swollen or tender joints, Likert score, VAS and DAS28 and the expression of PD-1 on CD4+ and CD8+ T cells. However, in our group of psoriatic patients with arthritis the mean values of both the VAS and DAS28 were lower than those reported by Peled et al. [14] (VAS $40.0 \pm 25.0$ vs. $46 \pm 5.5$ and DAS28 $3.2 \pm 1.1$ vs. $3.6 \pm 0.4$ ), whereas PASI was higher in our patients $(15.0 \pm 11.5)$ than in the studied group of Peled et al. [14] $(8.1 \pm 5.7)$. Similarly, the authors did not find any correlation between the level of PD-1 expressing T cells and ESR, CRP or PASI in the PSA patients. Moreover, in our study, PASI, BSA, PGA, NAPSI, and DLQI did not significantly differ between the psoriatic patients with arthritis and without arthritis. Therefore, we suspect that some other factors, e.g. genetic, might influence expression of the negative co-stimulators of T cells in psoriasis, thereby resulting in various severity and different clinical presentation of the disease.

The studies which provide evidence that the Th17 cell cytokines are promoters of osteoclastogenesis via induction of the receptor activator of the NF- $\mathrm{B}$ ligand (RANKL) give grounds to postulate that the role of decreased PD-1 expression in Th17 cytokine/RANKL axis activation is a possible explanation of PSA development [15]. In our previous study we observed that the osteoprotegerin, which is an important osteoclastogenesis inhibitor, to SRANKL ratio was significantly lower in the PSA than in the psoriatic patients without arthritis [16].

Although activation and hyperactivity of Th1 and Th17 in joint inflammatory diseases is well known, the results of the studies on the role of PD-1 in progression of arthritis and joint destruction in the pathogenesis of various arthropathies are inconclusive.
Earlier research into defective PD-1 expression in various rheumatologic diseases, i.e. PsA, rheumatoid arthritis (RA), systemic lupus erythematosus (SLE), and ankylosing spondylitis (AS), concerned the PD-1 gene polymorphisms [17-19]. Recently, studies have been focusing on the relationship of the expression levels of PD-1 and T cell activity and function.

Contrary to PSA, in RA increased PD-1 expression on T cells was found [20, 21].

However, in RA patients there were found high concentrations of serum and synovial SPD-1, a soluble form of PD-1, able to neutralize the increased PD-1 T cell expression. Therefore, even though PD-1 expression in RA is increased, it seems plausible that its capacity to regulate T cell activation is insufficient [20]. In SLE patients, some authors [22, 23] have also revealed increased expression of PD-1 on CD3+ and CD4+ T cells. Dolff et al. [23] in SLE patients did not observe any correlation between PD-1 expression as well as renal involvement and disease activity. However, in patients with AS, a disease which belongs to the same group as PsA, i.e. seronegative spondyloarthropathies (SpAs), the authors found decreased amounts of PD-1+ T cells, including follicular T cells, a subset of CD4+ T cells characterized by expression of PD-1 [24].

\section{Conclusions}

The findings reported in this paper are important for understanding the role of the negative co-stimulatory molecule PD-1 in T-cell activation in psoriasis with and without arthritis. Although the clinical presentations vary in many respects, psoriatic patients, both with and without arthritis, share some common inflammatory mediators and pathways. Thus, targeting the PD-1/PDL-1/Th17 axis/pathway may appear to be a potential therapeutic strategy in psoriatic patients.

\section{Acknowledgments}

The publication fee was covered by DS462/2016.

\section{Conflict of interest}

The authors declare no conflict of interest.

\section{References}

1. Nikamo P, Lysell J, Ståhle M. Association with genetic variants in the IL-23 and NF-kappaB pathways discriminates between mild and severe psoriasis skin disease. I Invest Dermatol 2015; 135: 1969-76.

2. Karczewski J, Dobrowolska A, Rychlewska-Hańczewska A, Adamski Z. New insights into the role of T cells in pathogenesis of psoriasis and psoriatic arthritis. Autoimmunity 2016; 49: 435-50.

3. Sibaud V, Meyer N, Lamant L, et al. Dermatologic complications of anti-PD-1/PD-L1 immune checkpoint antibodies. Curr Opin Oncol 2016; 28: 254-63. 
4. Bonigen J, Raynaud-Donzel C, Hureaux J, et al. Anti-PD1-induced psoriasis. A study of 21 patients. J Eur Acad Dermatol Venereol 2016; 31: e254-7.

5. Kato Y, Otsuka A, Miyachi Y, Kabashima K. Exacerbation of psoriasis vulgaris during nivolumab for oral mucosal melanoma. J Eur Acad Dermatol Venereol 2016; 30: e89-91.

6. Carter LL, Leach MW, Azoitei ML, et al. PD-1/PD-L1, but not PD-1/PD-L2, interactions regulate the severity of experimental autoimmune encephalomyelitis. J Neuroimmunol 2007; 182: 124-34.

7. Scarpa R, Ayala F, Caporaso N, Olivieri I. Psoriasis, psoriatic arthritis, or psoriatic disease? J Rheumatol 2006; 33: 210-2.

8. Kurt de Vlam. Immunopathology of the psoriatic arthritis musculoskeletal lesions. In: Psoriatic Arthritis and Psoriasis. Adebajo A, Boehncke WH, Gladman DD, Mease PJ (eds). Springer International Publishing Switzerland 2016; 53-60.

9. Chimenti MS, Ballanti E, Perricone C, et al. Immunomodulation in psoriatic arthritis: focus on cellular and molecular pathways. Autoimmun Rev 2013; 12: 599-606.

10. Ciocon DH, Kimball AB. Psoriasis and psoriatic arthritis: separate or one and the same? Br J Dermatol 2007; 157: 850-60.

11. Nakae S, Nambu A, Sudo K, Iwakura Y. Suppression of immune induction of collagen-induced arthritis in IL-17-deficient mice. J Immunol 2003; 171: 6173-7.

12. Greenwald RJ, Freeman GJ, Sharpe AH. The B7 family revisited. Annu Rev Immunol 2005; 23: 515-48.

13. Dulos J, Carven GJ, van Boxtel SJ, et al. PD-1 blockade augments Th1 and Th17 and suppresses Th2 responses in peripheral blood from patients with prostate and advanced melanoma cancer. J Immunother 2012; 35: 169-78.

14. Peled M, Strazza M, Azoulay-Alfaguter I, et al. Analysis of programmed death-1 in patients with psoriatic arthritis. Inflammation 2015; 38: 1573-9.

15. Paine A, Ritchlin C. Bone remodeling in psoriasis and psoriatic arthritis: an update. Curr Opin Rheumatol 2016; 28: 66-75.

16. Bartosińska J, Michalak-Stoma A, Juszkiewicz-Borowiec M, et al. The assessment of selected bone and cartilage biomarkers in psoriatic patients from Poland. Mediators Inflamm 2015; 2015: 194535.

17. Yang M, Zou Y, Bai Y, Li M. The programmed cell death 1 gene polymorphisms (PD 1.3 G/A, PD 1.5 C/T and PD 1.9 C/T) and susceptibility to ankylosing spondylitis: a meta-analysis. J Orthop Sci 2015; 20: 55-63.

18. Prokunina L, Padyukov L, Bennet A, et al. Association of the PD-1.3A allele of the PDCD1 gene in patients with rheumatoid arthritis negative for rheumatoid factor and the shared epitope. Arthritis Rheum 2004; 50: 1770-3.

19. Liu JL, Zhang FY, Liang YH, et al. Association between the PD1.3A/G polymorphism of the PDCD1 gene and systemic lupus erythematosus in European populations: a meta-analysis. J Eur Acad Dermatol Venereol 2009; 23: 425-32.

20.Liu C, Jiang J, Gao L, et al. Soluble PD-1 aggravates progression of collagen-induced arthritis through Th1 and Th17 pathways. Arthritis Res Ther 2015; 17: 340.

21. Wan B, Nie H, Liu A, et al. Aberrant regulation of synovial $T$ cell activation by soluble costimulatory molecules in rheumatoid arthritis. J Immunol 2006; 177: 8844-50.

22. Liu MF, Weng CT, Weng MY. Variable increased expression of program death-1 and program death-1 ligands on peripheral mononuclear cells is not impaired in patients with systemic lupus erythematosus. J Biomed Biotechnol 2009; 2009: 406136.

23. Dolff S, Quandt D, Feldkamp T, et al. Increased percentages of PD-1 on CD4+ T cells is associated with higher INF-gam- ma production and altered IL-17 production in patients with systemic lupus erythematosus. Scand I Rheumatol 2014; 43: 307-13.

24. Bautista-Caro MB, Arroyo-Villa I, Castillo-Gallego C, et al. Decreased frequencies of circulating follicular helper $T$ cell counterparts and plasmablasts in ankylosing spondylitis patients naïve for TNF blockers. PLoS One 2014; 9: e107086. 